\title{
Tecnura
}

\section{Sistema de control domótico de bajo costo: un respaldo a la generación ecológica de energía eléctrica en Colombia}

\author{
Low cost home automation system: \\ a support to the ecological electricity generation in Colombia
}

\author{
Elmer Alejandro Parada Prieto ${ }^{1}$, Mario Joaquín Illera Bustos² \\ Sergio Basilio Sepúlveda Mora ${ }^{3}$, Dinael Guevara Ibarra ${ }^{4}$, Byron Medina Delgado ${ }^{5}$
}

Fecha de recepción: 4 de febrero de 2015

Fecha de aceptación: 15 de mayo de 2016

Cómo citar: Parada Prieto, E. A., Illera Bustos, M. J., Sepúlveda Mora, S. B., Guevara Ibarra, D., \& Medina Delgado, B. (2016). Sistema de control domótico de bajo costo: un respaldo a la generación ecológica de energía eléctrica en Colombia. Revista Tecnura, 20(49), 120-132. doi: 10.14483/udistrital.jour.tecnura.2016.3.a08

\section{RESUMEN}

Contexto/objetivo: En Colombia, el consumo de energía eléctrica residencial representa aproximadamente el $40 \%$ de la demanda nacional, por lo tanto es relevante incursionar en alternativas que reduzcan este consumo. El objetivo de este trabajo fue elaborar un prototipo domótico de control de luminarias de una vivienda para el uso eficiente de la energía.

Método: El sistema consta de módulos de control autónomos y un módulo gestor de información; el módulo de control regula las luminarias usando un microcontrolador y un sensor de presencia, e intercambia datos a través de un transceptor de radio frecuencia; el módulo gestor permite el acceso a los módulos de control mediante una interfaz Web. El prototipo fue implementado en una vivienda en la ciudad de San José de Cúcuta, Colombia, por un periodo de sesenta días.
Resultados: La aplicación del sistema disminuyó el consumo total de la vivienda en un 3,75\%, con una unidad tipificada de -1,93 obtenida del análisis estadístico.

Conclusiones: Se concluye que el prototipo es de bajo costo en comparación con tecnologías similares disponibles en los mercados nacional e internacional, y que reduce el desperdicio de energía eléctrica producto de los hábitos de consumo de los residentes de la vivienda del caso de estudio.

Financiamiento: Universidad Francisco de Paula Santander.

Palabras clave: Domótica, interfaz Web, microcontrolador, Raspberry Pi.

\section{ABSTRACT}

Context/Objective: In Colombia, consumption of residential electricity accounts for about $40 \%$ of the national demand; therefore, alternatives to reduce

Ingeniero Electrónico. Ingeniero biomédico de la Clínica Medical Duarte. San José de Cúcuta, Colombia. Contacto: alejandro.parada.024@ gmail.com

Ingeniero Electrónico, ingeniero Instrumentista de Hyundai Engineering Co. San José de Cúcuta, Colombia. Contacto: mario.illera.b@gmail.com Ingeniero Electrónico, master of Science in Electrical and Computer Engineering. Docente de la Universidad Francisco de Paula Santander. San José de Cúcuta, Colombia. Contacto: sergio.sepulveda@ufps.edu.co

Ingeniero Eléctrico, especialista en Teleinformática, magíster en Ingeniería Electrónica, doctor en ingeniería. Docente de la Universidad Francisco de Paula Santander. San José de Cúcuta, Colombia. Contacto: dinaelgi@ufps.edu.co

Ingeniero Electrónico, magíster en Ingeniería Electrónica. Docente de la Universidad Francisco de Paula Santander. San José de Cúcuta, Colombia. Contacto: byronmedina@ufps.edu.co 
this consumption are needed. The goal of this study was to develop a home automation prototype to control the illumination of a household and to foster the efficient use of energy.

Method: The system consists of independent control modules and an information manager module; the control module regulates the luminaires using a microcontroller and a presence sensor, and exchanges data by means of a radio frequency transceiver; the manager module allows the access to the control modules from a Web interface. The prototype was implemented in a household located in the city of San José de Cúcuta, Colombia, during a 60 days period.
Results: The operation of the system diminished the total electricity consumption by $3,75 \%$, with a $z$-score of $-1,93$ that was obtained from the statistical analysis.

Conclusions: We concluded that the prototype is inexpensive in comparison to similar technologies available in the national and international markets, and it reduces the waste of electrical energy due to the consumption habits of the residents in the case study.

Keywords: Home automation, microcontroller, Raspberry Pi, Web interface.

\section{INTRODUCCIÓN}

En el sector eléctrico colombiano predomina la generación de energía hidráulica. Gracias a las particularidades de su geografía, como la abundante disponibilidad de fuentes hídricas y el variado relieve, la producción de electricidad en el país es más limpia -en comparación con otros que utilizan gas y petróleo-, con lo cual se mantienen bajas emisiones de gases de efecto invernadero. Aunque esto representa una gran ventaja ecológica, la producción de este tipo de energía es sensible a los cambios climáticos. En épocas de Iluvia, cuando los embalses funcionan a toda su capacidad, las hidroeléctricas Ilegan a cubrir hasta el $80 \%$ de la demanda nacional; sin embargo, en épocas de sequía, esta producción puede verse reducida considerablemente, Ilegando a aportar solo el 42,9 \% de esa generación (UPME, 2015). En estos periodos de baja generación hidráulica, el vacío debe ser cubierto por las termoeléctricas, las cuales, al quemar combustibles fósiles, pueden llegar a liberar todo el volumen de gases nocivos evitado por la generación limpia de energía.

Para reducir este impacto negativo de las épocas de sequía en las emisiones de gases es importante trabajar en mecanismos que logren unir esfuerzos en todos los ámbitos para generar un desarrollo sostenible del sector desde dos perspectivas diferentes: generación y consumo (Pinzón, Santamaría y Corredor, 2014; Gualteros, Ortiz, Sanabria y Sabogal, 2011). En el área de la generación ya se han realizado estudios que proponen utilizar otros tipos de energía disponibles en temporadas de bajas precipitaciones, como la energía solar (Gonzalez et al., 2012; Hernández, Díaz y Gordillo, 2009; Berrío y Zuluaga, 2014; Hernández, Cortés, Balaguera y Urueña, 2014) y la energía eólica (Caro et al., 2015; Isaac et al., 2010; Amaya, Saavedra y Arango, 2014), para generar energía eléctrica ecológica que respalde a la generación hidráulica.

Con respecto al consumo, en la sociedad colombiana cada vez es mayor la demanda energética por parte de las grandes ciudades, dando lugar a una crisis en este sector (González, Ferro y Tarazona, 2014). Un incremento en la cantidad de habitantes, la construcción de nuevas urbanizaciones y el aumento en el número de aparatos eléctricos en el hogar, producto del gran avance tecnológico de los últimos años, han llevado a que en la última década el consumo residencial sea el destino del 40,6 \% de la producción energética total del país, siendo este sector el de mayor participación por encima del industrial y comercial.

Por esta razón, para reducir el consumo eléctrico en el país es necesario desarrollar un plan 
de impacto a gran escala, cuyo objetivo sea disminuir, en primera instancia, el consumo por parte del sector residencial. En algunas zonas del país se han comenzado a realizar esfuerzos para buscar alternativas tecnológicas eficaces para afrontar esta problemática (Tapia et al., 2013), pero en ocasiones no son lo suficientemente globales y flexibles para su aplicación (Céspedes, et al., 2012). Gracias al gran avance e impacto de la domótica en la actualidad, se proyecta como uno de los caminos más eficientes para cumplir con este objetivo (Longo, Roscia y Zaninelli, 2015), basado en el desarrollo de sistemas de control de bajo costo que facilitan su implementación en la mayoría de los hogares colombianos (Quintana, Pereira y Vega, 2015), generando no solo un consumo sostenible de la energía sino una conciencia responsable sobre la participación de cada uno de los hogares del país en el tema.

Siendo la red de iluminación uno de los principales focos de desperdicio energético residencial en la actualidad (Carrion, 2013), y basados en previas investigaciones en este ámbito (García, Moctezuma y Villa, 2014); este trabajo presenta un sistema domótico que controla la iluminación de una vivienda, mediante módulos de control autónomos y un módulo gestor de información. El prototipo es escalable, de bajo consumo de energía, de bajo costo, y contribuye con la solución de la problemática energética mencionada. En primer lugar, se muestra la arquitectura del sistema con los distintos componentes y su interacción; posteriormente se presenta una evaluación del sistema en condiciones reales, y por último se plantean las conclusiones.

\section{METODOLOGÍA}

La principal función del sistema domótico es realizar un control eficiente de la red de iluminación de la vivienda, por lo que se hace necesario monitorizar todos los elementos lumínicos de esta. La vivienda utilizada para esta investigación posee seis focos distribuidos en cuatro habitaciones, una sala y una cocina, por lo que se requiere la implementación de seis módulos de control para evaluar y controlar los hábitos de consumo de sus habitantes.

A pesar de que los sistemas domóticos tradicionales utilizan líneas físicas de conexión, se opta por un protocolo de comunicación inalámbrica para la interacción de los dispositivos entre sí, como lo sugieren Nedelcu et al. (2009) y Vera, Barbosa y Pabón (2015). Lo anterior otorga tres características importantes al sistema definidas por Apolloni et al. (2010): escalabilidad completa para la integración de nuevos dispositivos, control autónomo para la toma de decisiones y plena operatividad por parte del usuario ante cualquier evento en el hogar. Estas dos últimas, aunque pueden presentarse en sistemas domóticos tradicionales, aumentan su eficiencia con el uso de la tecnología inalámbrica que ofrece mejoras en la transmisión de datos, acceso a lugares en condiciones complejas, bajo costo de implementación y de consumo energético (Nguyen, Nguyen, Kien, Cuong y Nguyen, 2015; Hernández et al., 2010), entre otras muchas ventajas que se pueden explotar en diferentes tipos de aplicaciones (Filho, Ueyama, Faiçal, Guidoni y Villas, 2015; Leal, Albarracín y Ortega, 2009; Claros y Casanova, 2009).

En estas condiciones, una topología óptima para el sistema es una arquitectura distribuida (una de las dos formas de implementación basadas en la ubicación de la inteligencia del sistema), que permite agrupar toda la información en una central de gestión para almacenar y presentar datos que le dan al usuario la capacidad de supervisar y controlar todo el sistema, sin eliminar totalmente la autonomía de los módulos ubicados en la diferentes zonas de control (Herrera, 2005). Cada módulo es capaz de capturar información proveniente de los sensores, analizarla y ejecutar la acción pertinente a través de los actuadores, mientras el elemento central no indique lo contrario. Así, cada parte del sistema trabaja de acuerdo con las necesidades de cada sector, con una dependencia parcial de las 
decisiones del usuario. Un diagrama general de la arquitectura del sistema se muestra en la figura 1.

De esta forma, existe un centro de operaciones, o central de gestión de información, con una serie de módulos interconectados inalámbricamente que controlan los focos lumínicos de toda la vivienda; la central de gestión implementa una plataforma de control que funciona como puente entre el usuario y el sistema. Teniendo en cuenta que la interoperabilidad es uno de los principales problemas de los sistemas domóticos en la actualidad, todos los componentes se diseñaron para que se acoplen de la mejor forma posible y darle, como se mencionó, una mejor escalabilidad al sistema en el futuro (Bonino, Castellina y Corno, 2009). A continuación se describe cada uno de los módulos del sistema.

\section{Módulos de control: diseño del hardware}

Para lograr la autonomía de cada módulo de control se integran los siguientes elementos para ejecutar las acciones de monitorización y control:

En primer lugar, un detector de movimiento, el sensor HC-SR501, que emplea un sensor PIR (passive infrared sensor) para medir la radiación infrarroja emitida por los objetos en su campo de visión, que permite detectar la presencia de personas a través de los cambios de temperatura en el lugar.
Como segundo elemento se integró un actuador encargado de realizar la acción final de control, en este caso encendiendo o apagando el bombillo de la habitación. El dispositivo escogido para realizar esta función es el triac BT134600 que puede soportar corrientes de hasta 4 A y voltajes de $600 \mathrm{~V}$, características que sobrepasan ampliamente las necesidades eléctricas de los bombillos instalados en la residencia, previniendo de esta manera posibles fallas durante su funcionamiento continuo.

El tercer elemento que conforma cada módulo de control es el módulo de comunicación. Para implementar un canal de comunicación inalámbrica es necesario contar con módulos de radiofrecuencia que soporten el tráfico de datos del sistema. El módulo nRF24L01 es un transceptor inalámbrico que opera a una frecuencia de $2,4 \mathrm{GHz}$ y ofrece una gran flexibilidad de operación, ya que permite utilizar hasta 128 canales diferentes para la comunicación, además de 255 direcciones por cada canal que pueden ser asignadas y configuradas de manera sencilla a través de un microcontrolador mediante la interfaz periférica serial (SPI, serial peripheral interface). Este módulo es el encargado de recibir y transmitir datos, desde y hacia la central de gestión ubicada en el módulo central, lo que posibilita, por una parte, recibir las órdenes dadas por el usuario, y por otra, enviar la información del estado del módulo.

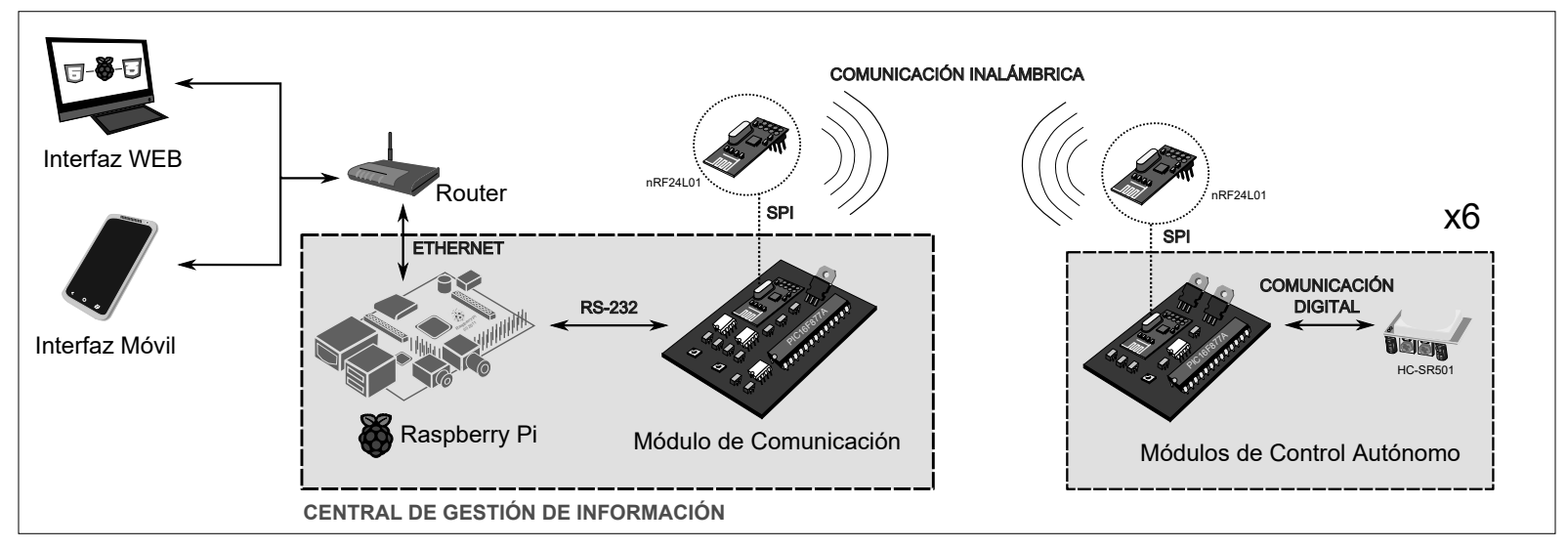

Figura 1. Arquitectura general del sistema

Fuente: elaboración propia. 
Por último, para llevar a cabo las funciones de control se seleccionó el microcontrolador PIC16F877A de microchip. Este dispositivo ofrece un gran número de funciones que se adaptan a las necesidades del módulo de control, como entradas y salidas digitales, interrupciones internas y externas, y un módulo de comunicación SPI. El microcontrolador lleva a cabo varias funciones dentro del circuito, es el encargado de comprobar el estado de la salida digital del sensor, procesar esta información y realizar la acción pertinente a través del actuador. Controla además la comunicación con el módulo de radiofrecuencia a través del protocolo SPI y realiza ajustes en los tiempos de activación del actuador.

\section{Módulos de control: diseño del firmware}

Para que el microcontrolador pueda llevar a cabo las funciones de control y comunicación con el módulo de radiofrecuencia, por medio del protocolo SPI, fue necesario desarrollar un firmware que reuniera las instrucciones necesarias para su correcto funcionamiento. Para tal fin se implementaron una serie de funciones que recogen y analizan los datos provenientes del sensor y de la central de gestión de información, y ejecutan la acción pertinente para cada caso.

El microcontrolador evalúa el estado del sensor de movimiento, para encender o apagar la bombiIla, de acuerdo con la presencia o no de una persona en su lugar de monitorización. Al detectar movimiento, no solo se enciende la red de iluminación, sino que se inicia un temporizador que almacena su tiempo de activación.

El temporizador posee unos límites de operación, que al alcanzarse ejecutan acciones según el estado del actuador. Si este se encuentra desactivado, este tiempo se acumula en una variable que luego es almacenada como información de las horas, minutos y segundos que ha estado apagado el bombillo. Si el actuador está activado, este tiempo se acumula en una segunda variable similar que lleva el registro del tiempo que el bombillo ha estado encendido. Se realiza además una comparación con el tiempo límite que debe estar activado el actuador, así, si estos tiempos son iguales, el actuador es desactivado. Si el sensor detecta un movimiento mientras el bombillo está encendido, esta segunda variable es reiniciada.

Por último, los módulos de control instalados en la vivienda son configurados para tener la capacidad de establecer una comunicación half-duplex con la central de gestión de información, ya sea para recibir órdenes provenientes del usuario o para transmitir la información de su estado actual, de una forma segura y eficaz para no generar retrasos en las acciones de control.

\section{Optimización del funcionamiento}

Con el fin de prevenir el desperdicio de energía por parte del sistema y mejorar la experiencia de los usuarios, se incorporó en el firmware del microcontrolador la capacidad de ajustar autónomamente el tiempo límite que debe estar activado el actuador. De esta manera se evita que el bombillo esté encendido por un tiempo mayor o menor al establecido por las necesidades del usuario.

Esto se logra mediante el análisis del tiempo que transcurre entre movimientos de la persona que ocupa la habitación. Cada vez que se activa el sensor, el microcontrolador empieza a llevar un conteo del tiempo a través de una variable; si un nuevo movimiento es detectado antes de que esta variable llegue al límite permitido, su valor es almacenado en un vector y esta reinicia su cuenta. Este proceso se repite para almacenar un número determinado de datos y cuando el vector se llena se procesa la información guardada. De este procedimiento el módulo obtiene su nuevo límite de tiempo de activación del actuador y se ajusta a esta nueva configuración.

De la lógica de este proceso se puede concluir que el nuevo tiempo límite tiende a ser menor que el inicial, y así se podría llegar a presentar la situación de que sea tan pequeño que el sistema, en lugar de aportar al confort de los usuarios, se convierta 
en una molestia al apagar las luces cuando todavía haya alguien en la habitación. Para evitar este inconveniente, el microcontrolador también analiza el tiempo que transcurre entre el apagado automático y la detección de un nuevo movimiento. Si este es lo suficientemente extendido, indica que la persona efectivamente abandonó la habitación y el valor del tiempo límite es el adecuado; por otra parte, si este tiempo es muy corto, indica que la persona continuaba en la habitación y el tiempo límite debería ser mayor al actual. Si esta última situación se repite un determinado número de veces, el módulo de control automáticamente incrementa el límite de tiempo de encendido y se ajusta a esta nueva configuración. Así, el módulo se adapta a los hábitos y costumbres de cada uno de sus usuarios de forma permanente.

\section{Central de gestión de información}

Como se mencionó, a pesar de la autonomía de cada módulo de control, el sistema ofrece al usuario la opción de supervisar y modificar el estado de todos los componentes del sistema en cualquier momento. Por tal razón, a la hora de presentar la información del estado de la vivienda al usuario final, es necesario contar con un equipo servidor que se encargue de realizar dos funciones principales: por una parte, comunicarse con los módulos instalados en las diferentes habitaciones y almacenar los datos recibidos; y por otra, organizar la información en una interfaz para que el usuario pueda acceder a ella de manera sencilla y eficaz. Para lograr estos objetivos, la central de gestión de información integra dos dispositivos: un módulo de comunicación que se encarga de transmitir y recibir información, y un ordenador de placa reducida con la capacidad de alojar un servidor web donde se presentan de manera ordenada e interactiva los datos almacenados. El diagrama de flujo que representa la comunicación entre el módulo de control y el ordenador de placa reducida se muestra en la figura 2 .
Con el propósito de implementar una interfaz gráfica para que el usuario acceda fácilmente a los datos y al control del sistema, es necesario analizar las diferentes posibilidades y elegir la más adecuada. Uno de los objetivos de este trabajo es brindar al usuario final la posibilidad de acceder al sistema a través de un smartphone. Para lograr esto, hay dos opciones que se pueden implementar: el desarrollo de una aplicación orientada al sistema operativo del smartphone, o el desarrollo de una aplicación web (IBM Corporation, 2012). La segunda opción ofrece una gran ventaja y es la portabilidad que adhiere al sistema, ya que al estar alojada en un servidor web, esta puede ser consultada desde cualquier dispositivo con acceso a internet, como ordenadores, tabletas y smartphones. La aplicación web fue entonces la opción elegida para implementar la interfaz de usuario, y para alojar el servidor web se escogió una Raspberry $\mathrm{Pi}$, un ordenador de placa reducida de bajo costo que, gracias a sus notables características, ofrece grandes posibilidades a la hora de desarrollar aplicaciones que requieran acceso a través de internet (Computing at School, 2012).

En la Raspberry Pi se configuró el servidor HTTP Apache, un servidor web de código libre, que aloja los archivos necesarios para el correcto funcionamiento de la interfaz web. Esta última ofrece al usuario la posibilidad de controlar de manera interactiva el estado de las luces en el hogar. Para ello implementa Ilamadas a través de $p h p$, a programas desarrollados en lenguaje Python para traducir las órdenes y comunicarlas a los módulos de control. La Raspberry Pi se encarga además de activar y desactivar los sensores automáticamente, esto mediante un algoritmo desarrollado en Python en el cual se indica la hora en la que se debe realizar cada acción; cuando se ejecuta el comando para desactivar los sensores, la Raspberry también envía una solicitud a los diferentes módulos para actualizar la información de su estado y esta es almacenada para su posterior visualización por parte del usuario. 


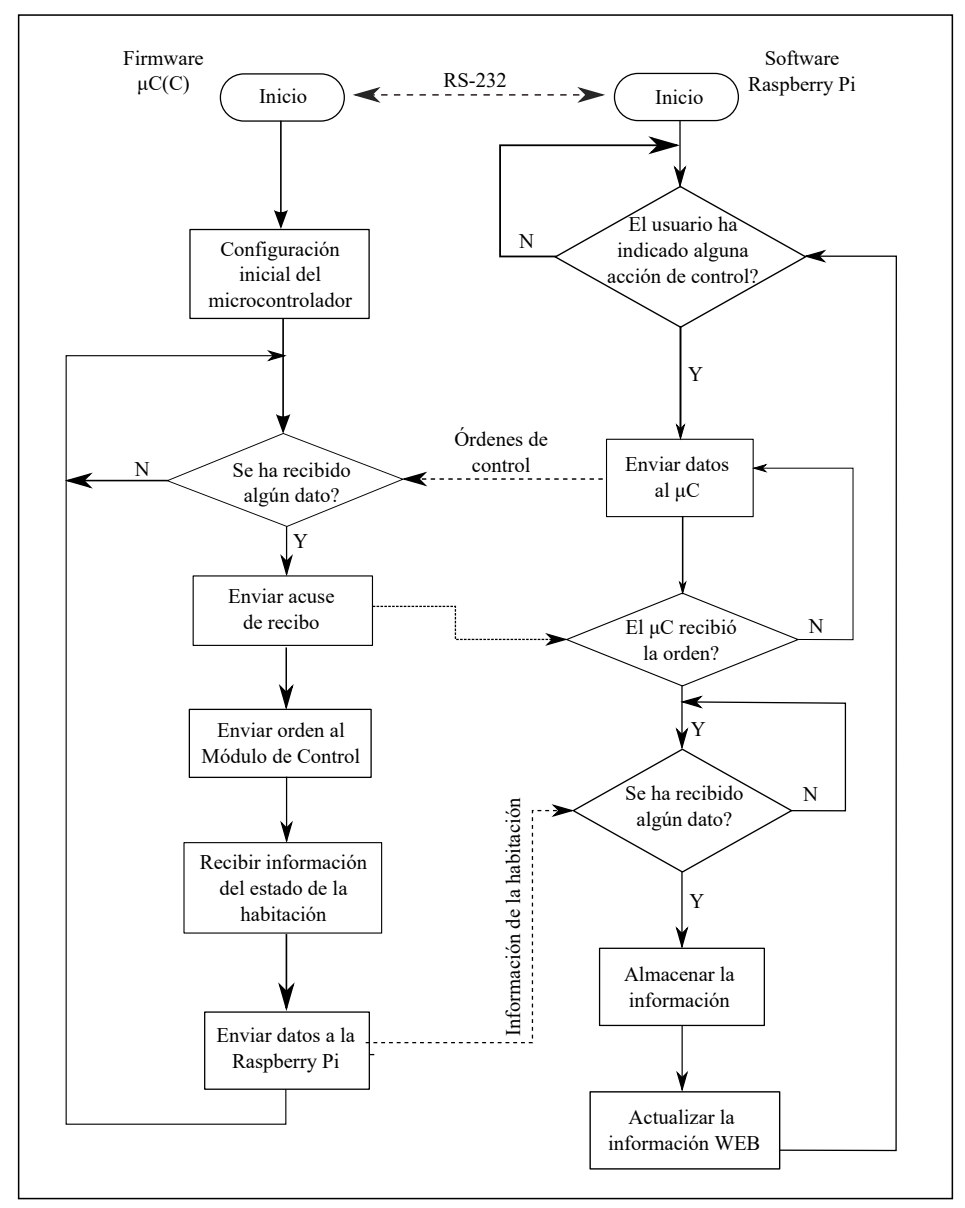

Figura 2. Algoritmos de control

Fuente: elaboración propia.

Para establecer una comunicación estable y segura con los módulos de control se diseñó una tarjeta con la capacidad de transmitir comandos, recibir datos y almacenar la información de manera ordenada. El hardware del módulo de comunicación está conformado por dos componentes principales: un módulo de radiofrecuencia $\mathrm{nR}$ F24L01 que se emplea para establecer una comunicación inalámbrica half-duplex con los módulos de control, y un microcontrolador PIC16F877A que gestiona los diferentes protocolos de comunicación empleados para intercambiar información con el ordenador de placa reducida y el módulo de radiofrecuencia, que almacena y gestiona los datos obtenidos. El intercambio de información entre el microcontrolador y el ordenador de placa reducida se realiza a través del protocolo serial RS232 (Recommended Standard 232), utilizado por su fácil implementación y la confianza que ha mostrado en proyectos similares (Dobrescu, 2014).

\section{Interfaz web}

En la interfaz de usuario se puede observar el estado de los bombillos de cada una de las habitaciones, enviar órdenes para encenderlos o apagarlos, y consultar estadísticas de consumo. Esta aplicación fue desarrollada en HTML (hypertext markup language) empleando hojas de estilo en cascada o CSS (cascading style sheets) a través de las cuales se pueden modificar características de los elementos que conforman la página entre otras 
propiedades que aportan a mejorar su contenido visual. Ahora, para que la aplicación sea realmente útil, debe permitir al usuario interactuar con sus diferentes elementos, para ello es necesario contar con un algoritmo que tenga la capacidad de interpretar las órdenes, procesarlas y ejecutar las acciones indicadas dependiendo del caso. Esto se logra a través de JavaScript, un lenguaje de programación que permite crear algoritmos con gran capacidad para procesar información del entorno web y tomar decisiones (Gauchat, 2012). Estas herramientas dan a la aplicación gran versatilidad y permiten llevar a cabo las funciones de visualización y control requeridas por el sistema domótico.

\section{RESULTADOS}

El objetivo principal de esta investigación era desarrollar un sistema de control domótico con la capacidad de reducir el consumo energético de una vivienda a través de la gestión de su sistema de iluminación. Para evaluar su desempeño, una vez instalado, el sistema fue puesto a prueba por un periodo de 60 días en los cuales se registró de forma diaria el consumo de energía eléctrica de la vivienda para valorar posteriormente su reducción o incremento por el aporte del sistema de control. Las especificaciones de potencia de los módulos de control y la central de gestión de información, se detallan en la tabla 1.

Tabla 1. Características de potencia de los dispositivos

\begin{tabular}{llll}
\hline \multirow{2}{*}{ Dispositivo } & \multicolumn{3}{c}{ Características } \\
\cline { 2 - 4 } & $\begin{array}{c}\text { Voltaje } \\
(\mathbf{V})\end{array}$ & $\begin{array}{c}\text { Corriente } \\
(\mathbf{m A})\end{array}$ & $\begin{array}{c}\text { Potencia } \\
(\mathbf{W})\end{array}$ \\
\hline Módulo de control & 12 & 10 & 0,12 \\
\hline Central de gestión & 5 & 560 & 2,80 \\
\hline
\end{tabular}

Fuente: elaboración propia.

Antes de analizar los datos obtenidos se consultó el historial de consumo de la vivienda, tomando como base la información registrada en las facturas de la empresa que presta el servicio de suministro de energía eléctrica.

La figura 3 ilustra el consumo de la vivienda en kWh para el periodo comprendido entre abril y septiembre de 2015.

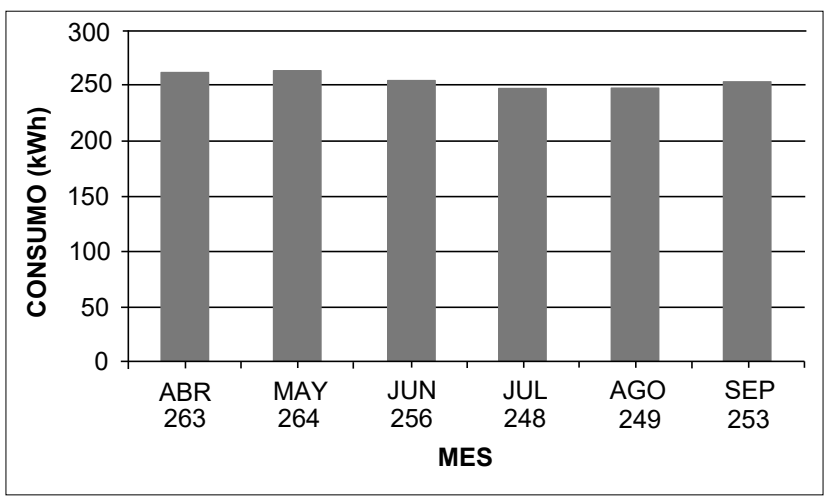

Figura 3. Consumo de la residencia abril-septiembre de 2015

Fuente: elaboración propia.

Con base en la ecuación (1) y los valores de la figura 3 se obtuvo el consumo promedio de la vivienda bajo condiciones normales de 255,5 kWh.

$$
\bar{x}=\frac{1}{n} \sum_{i=1}^{n} x_{i}
$$

La prueba del dispositivo se dividió en dos etapas de 30 días cada una, tomando como referencia el periodo analizado en la figura 3, con el fin de obtener una comparación fiable del consumo del sistema. En la primera etapa se registró el consumo de la vivienda con los módulos de control instalados sin la capacidad de ajustar autónomamente el tiempo de encendido del bombillo y sin la central de gestión de información; de este modo, el sistema solo realizaba la acción de control sobre las luces encendiéndolas por un periodo fijo cada vez que el sensor detectaba un movimiento. Para realizar el registro del consumo durante la primera etapa se tomaron lecturas diarias del contador eléctrico instalado en la residencia, realizando un análisis sobre el comportamiento diario de la red. 
Para los primeros treinta días de prueba el consumo diario máximo promedio fue de 9,6 kWh registrado el día 7, y el mínimo fue de 7,6 kWh registrado el día 21. El consumo energético total fue de 251,9 kWh con un promedio de 8,4 kWh por día. De los resultados de la primera etapa se puede observar que el consumo se mantuvo por debajo del promedio de los meses anteriores, con una disminución del 1,33\% equivalente a 3,4kWh, lo cual quiere decir que el funcionamiento continuo del sistema no aporta al gasto eléctrico del hogar, sino que permite mantener un consumo eléctrico estable de la vivienda.

En el segundo periodo de prueba se modificó el firmware del sistema para incluir, por una parte, la capacidad de ajustar autónomamente el tiempo de encendido, y por otro lado la central de gestión de información. Para realizar el registro del consumo durante esta segunda etapa, se realizó el mismo proceso aplicado a la primera, ilustrando los resultados en la figura 4, realizando un contraste entre los dos periodos de prueba.

En esta etapa el consumo diario máximo fue de $8,7 \mathrm{kWh}$ registrado el día 10, y el mínimo fue de $7,2 \mathrm{kWh}$ registrado el día 12 . El consumo energético total fue de $242,3 \mathrm{kWh}$ con un promedio de 8,08 kWh por día. A partir de la ecuación (2) y de los datos de la figura 4, se determina la variabilidad del consumo de energía eléctrico en la vivienda antes de implementar el sistema domótico, correspondiente a 6,83 kWh.

$$
\sigma=\sqrt{\frac{1}{n-1} \sum_{i=1}^{n}\left(\mathrm{x}_{i}-\bar{x}\right)^{2}}
$$

Teniendo en cuenta lo anterior, se puede determinar el número de desviaciones típicas entre los resultados obtenidos con el sistema y el promedio del consumo de la vivienda sin él, por medio de la ecuación (3).

$$
\begin{gathered}
Z=\frac{x-\bar{x}}{\sigma} \\
Z=\frac{242,3 k W h-255,2 k W h}{6,83 k W h} \\
Z=-1,93
\end{gathered}
$$

Esta variable calculada se conoce también como unidad tipificada. Es el número de desviaciones estándar que hay de diferencia entre el consumo con el sistema propuesto y el promedio anterior. El valor de -1,93 permite inferir que la reducción del consumo de energía eléctrica fue significativo y que no se debe a un evento aleatorio. Estos datos demuestran que las mejoras implementadas en el sistema contribuyeron a la disminución del consumo de energía eléctrica en la vivienda, a través de la gestión optimizada de su sistema de iluminación. La tabla 2 resume los resultados de las etapas de prueba realizadas.

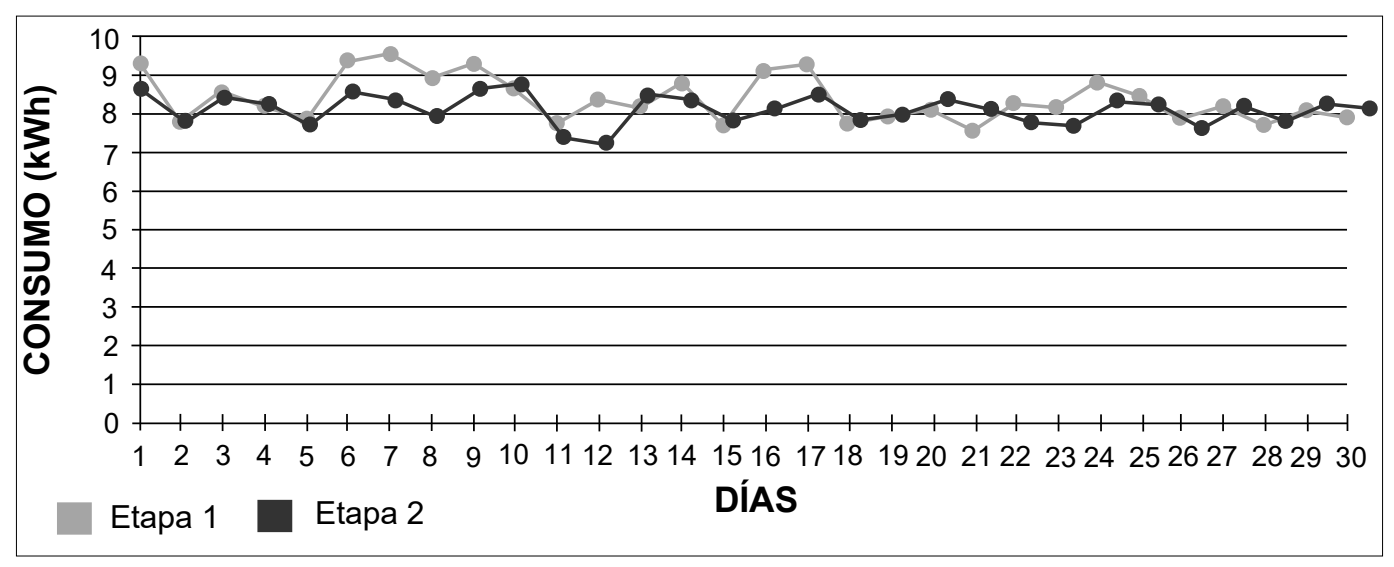

Figura 4. Consumo eléctrico de la vivienda: etapa 1 vs. etapa 2

Fuente: elaboración propia. 
Tabla 2. Resumen del consumo de la vivienda

\begin{tabular}{|c|c|c|c|c|c|}
\hline \multirow{2}{*}{$\begin{array}{c}\text { Etapa de } \\
\text { prueba }\end{array}$} & \multicolumn{3}{|c|}{ Consumo diario (kWh) } & \multirow{2}{*}{$\begin{array}{c}\text { Consumo promedio } \\
\text { mensual }(\mathbf{k W h})\end{array}$} & \multirow{2}{*}{$\begin{array}{l}\text { Reducción promedio } \\
(\mathrm{kWh})^{*}\end{array}$} \\
\hline & Máx. & Mín. & Promedio & & \\
\hline Etapa 1 & 9,6 & 7,6 & 8,40 & 251,9 & 3,4 \\
\hline Etapa 2 & 8,7 & 7,2 & 8,08 & 242,3 & 13,2 \\
\hline
\end{tabular}

*Reducción promedio con respecto al consumo sin el sistema domótico.

Fuente: elaboración propia.

Por último, en la tabla 3 se realiza una comparación entre los principales elementos del sistema y algunas tecnologías análogas presentes en el mercado, con precios nacionales (I + D Electrónica, s.f.) e internacionales (eBay, s.f.) como lo indica Hincapie, Duarte y Sepúlveda (2015), donde se puede evidenciar que el sistema domótico presentado en esta investigación puede alcanzar una reducción de costos de entre el 70 y 85 \% (variables según los accesorios necesarios para su aplicación) con respecto a sistemas similares, representando así una alternativa de bajo costo para su implementación en diferentes sectores sociales del país.

\section{CONCLUSIONES}

El sistema de control domótico desarrollado tiene la capacidad de reducir el desperdicio de energía eléctrica producto de los hábitos de consumo de las personas que habitan en una vivienda, al realizar el control y gestión de su sistema de iluminación, con un ahorro de cerca del 3,75\% del consumo total, el cual es un valor significativo tomando en cuenta la variabilidad del consumo de meses anteriores (sigma $=6,83 \mathrm{kWh}$ ) y la unidad tipificada del consumo con el sistema domótico $(z=-1,93)$.

Tabla 3. Comparación de precios para diferentes dispositivos

\begin{tabular}{|c|c|c|c|c|}
\hline & Dispositivo & $\begin{array}{c}\text { Mercado } \\
\text { nacional (COP) }\end{array}$ & $\begin{array}{c}\text { Mercado } \\
\text { internacional (USD)* }\end{array}$ & $\begin{array}{c}\text { Porcentaje de ahorro } \\
\text { aprox. (COP) }\end{array}$ \\
\hline \multirow{3}{*}{$\begin{array}{l}\text { Dispositivos de comu- } \\
\text { nicación inalámbrica }\end{array}$} & nRF24L01 & 27.000 & 3,15 & \multirow{3}{*}{$70-80 \%$} \\
\hline & Zigbee Xbee S2 & 100.000 & 33,82 & \\
\hline & $\begin{array}{l}\text { GSM/GPRS shield Arduino } \\
\text { M95 }\end{array}$ & 150.000 & 25 & \\
\hline \multirow{3}{*}{$\begin{array}{l}\text { Dispositivos de control } \\
\text { y procesamiento de } \\
\text { datos }\end{array}$} & $\begin{array}{l}\text { Microcontrolador PIC- } \\
\text { 16F877A }\end{array}$ & 15.000 & 2,35 & \multirow{3}{*}{$80-85 \%$} \\
\hline & Arduino Uno R3 & 70.000 & 7,35 & \\
\hline & Netduino & 100.000 & 60,29 & \\
\hline \multirow{3}{*}{ Servidores } & Raspberry Pi & 150.000 & 38,82 & \multirow{3}{*}{$80 \%$} \\
\hline & FPGA Nexys 3 & 780.000 & 250 & \\
\hline & Ordenador personal & 700.000 & 230 & \\
\hline
\end{tabular}

*Los precios pueden variar de acuerdo al proceso de importación del equipo.

Fuente: elaboración propia. 
La arquitectura híbrida empleada en el diseño del prototipo da como resultado un sistema de bajo costo, en comparación con tecnologías similares disponibles en los mercados nacional e internacional; además, el sistema es fácil de implementar en diferentes niveles económicos del país, con una gran escalabilidad para la incorporación de nuevos módulos de control sin necesidad de implementar grandes modificaciones en la estructura del sistema, ni en la vivienda donde está instalado.

La capacidad de configuración autónoma de los módulos de control para ajustar los tiempos de encendido de las luminarias, otorga al sistema gran flexibilidad para adaptarse a las necesidades de consumo que tengan los usuarios. Este proceso a su vez optimiza la gestión del consumo de energía al prevenir los desperdicios que pueda generar el funcionamiento continuo del sistema.

Con base en la apreciación de los residentes de la vivienda del caso de estudio, la experiencia de interactuar con el sistema de control domótico generó un impacto social y ambiental, porque creó conciencia en ellos sobre la importancia de consumir responsablemente la energía eléctrica necesaria para sus actividades diarias.

\section{REFERENCIAS BIBLIOGRÁFICAS}

Amaya M., P.A.; Saavedra M., A.J. y Arango Z., E.I. (2014). A statistical analysis of wind speed distribution models in the Aburrá Valley, Colombia. CT\&FCiencia, Tecnología y Futuro, 5(5), 121-136.

Apolloni, B.; Bassis, S.; Galliani, G.L. y Valerio, L. (2010). Wireless Domotic: An Enabling Platform for Granular Intelligence. En: 2010 5th International Conference on Future Information Technology (FutureTech) (pp. 1-6). Busan, Corea del Sur. doi: 10.1109/FUTURETECH.2010.5482697

Berrío, L.H. y Zuluaga, C. (2014). Smart Grid y la energía solar fotovoltaica para la generación distribuida: una revisión en el contexto energético mundial. Ingeniería y Desarrollo, 32(2), 369-396. doi: 10.14482/inde.32.2.4957
Bonino, D.; Castellina, E. y Corno, F. (2009). Automatic domotic device interoperation. IEEE Transactions on Consumer Electronics, 55(2), 499-506. doi: 10.1109/TCE.2009.5174413

Caro, M.; Fonseca, J.; Jiménez, B.; Rodríguez, R. y Zapata, H. (2015). Integration of wind power plants into the Colombian power system. En: 2015 IEEE PES Innovative Smart Grid Technologies Latin America (ISGT LATAM) (pp. 264-269). Montevideo, Uruguay. doi: 10.1109/ISGT-LA.2015.7381165

Carrion, D. (2013). Energy saving model through lighting management systems for residences. En 2013 Workshop on Power Electronics and Power Quality Applications (PEPQA) (pp. 1-6). Bogotá, Colombia. doi: 10.1109/PEPQA.2013.6614955

Céspedes, R.; León, R.A.; Salazar, H.; Ruiz, M.E.; Hidalgo, R. y Mejía, D. (2012). An appraisal of the challenges and opportunities for the Colombia Inteligente Program implementation. En: 2012 IEEE Power and Energy Society General Meeting (pp. 1-6). San Diego, Estados Unidos. doi: 10.1109/ PESGM.2012.6345383

Claros L., R. y Casanova A., J.A. (2009). Sistema inalámbrico de adquisición de vibraciones orientado a la detección de movimientos sísmicos con tecnología Zigbee. ITECKNE, 6(1), 20-28. doi: 10.15332/iteckne.v6i1.292

Computing at School (diciembre de 2012). The Raspberry Pi Education Manual. Recuperado de: http:// pi.cs.man.ac.uk/download/Raspberry_Pi_Education_Manual.pdf

Dobrescu, L. (2014). Domotic embedded system. 2014 6th International Conference on Electronics, Computers and Artificial Intelligence (ECAI) (pp. 55-58). Bucarest, Rumania. doi: 10.1109/ ECAI.2014.7090180

eBay. (s.f.). Digilent Nexys 3 FPGA Board, Netduino Plus, Raspberry pi board. Recuperado de: http:// www.ebay.com/

Filho, G.P.R.; Ueyama, J.; Faiçal, B.S.; Guidoni, D.L. y Villas, L. A. (2015). ResiDI: An Intelligent Decision Platform for Residential Infrastructure Using Wireless Sensors and Actuators. 2015 XXXIII Brazilian Symposium on Computer Networks and 
Distributed Systems (SBRC) (pp. 237-245). Victoria, Brasil. doi: 10.1109/SBRC.2015.36

García G., J.; Moctezuma M., E.O. y Villa L., F.H. (2014). FPGA implementation of a smart home lighting control system. En 2014 IEEE Fourth International Conference on Consumer Electronics Berlin (ICCE-Berlin) (pp. 419-420). Berlín, Alemania. doi: 10.1109/ICCE-Berlin.2014.7034320

Gauchat, J. (2012). El gran libro de HTML5, CSS3 y Javascript. Barcelona, España: Macombo.

Gonzalez, D.; Ramos P., C.A.; Saavedra M., A.J.; Arango Z., E.I. y Carrejo, C.E. (2012). Modeling and control of grid connected photovoltaic systems. Revista de Facultad de Ingeniería Universidad de Antioquia, 62, 145-156.

González B., R.A.; Ferro E., R. y Tarazona B, G. (2014). Development of a uml framework for smart cities with forecasting electrical consumption in $\mathrm{Co}$ lombia. Revista Tecnura, 18(Edición especial doctorado), 109-123. doi: 10.14483/udistrital.jour. tecnura.2014.DSE1.a10

Gualteros, M.; Ortiz, D.; Sanabria, S. y Sabogal, J. (2011). Possibilities for CDM project implementation in the framework of the usage of renewable energy in Colombia: An evolutionary approach. En: 2011 IEEE PES Conference on Innovative Smart Grid Technologies (ISGT Latin America) (pp. 1-5). Medellín, Colombia. doi: 10.1109/ISGT-LA.2011.6083204

Hernández, J.; Díaz, N.L. y Gordillo, G. (2009). Design-dimensioning model for grid-connected photovoltaic systems. En: 2009 IEEE Electrical Power \& Energy Conference (EPEC) (pp. 1-5). Montreal, Canadá. doi: 10.1109/EPEC.2009.5420962

Hernández M., J.A.; Cortés B., A.F.; Balaguera C., D.A. y Urueña S., M.A. (2014). Aplicación de los sistemas fotovoltaicos conectados a la red: estado del arte. Revista Tecnura(Edición especial), 157-172. doi: 0.14483/udistrital.jour.tecnura.2014.SE1.a12

Hernández O., J.M.; Inzunza G., E.; Olguín T., J.E.; Soarez L., J.J.; Rousseau F., P.A. y Camargo W., C. (2010). Protocolo MAC aplicado a 802.15.4 eficiente en el consumo de energía. Ingeniería e investigación, 30(1), 71-74.
Herrera Q., L.F. (2005). Viviendas inteligentes (Domótica). Revista Ingeniería e Investigación, 25(2), 47-53. Hincapie, D. M.; Duarte, G.G. y Sepúlveda, S.B. (2015). Low-cost and Reliable Wireless Communication System for Monitoring a Photovoltaic Source. En: 2015 IEEE Colombian Conference on Communications and Computing (COLCOM) (pp. 1-5). Popayán, Colombia. doi: 10.1109/ ColComCon.2015.7152088

I + D Electrónica (s.f.). Arduino Uno R3, PIC16F877A, Raspberry Pi. Recuperado de: http://www.didacticaselectronicas.com/

IBM Corporation (abril de 2012). El desarrollo de aplicaciones móviles nativas, web o híbridas. Recuperado de: ftp://ftp.software.ibm.com/la/documents/gb/ commons/27754_IBM_WP_Native_Web_or_hybrid_2846853.pdf

Isaac, I.; González, J.; Areiza, J.; Biechl, H.; Cardona, H. y Lopez, G. (2010). Large scale integration of wind energy in Colombia: Electrical analysis-part I. En: 2010 IEEE/PES Transmission and Distribution Conference and Exposition: Latin America (T\&DLA) (pp. 231-238). Sao Paulo, Brasil. doi: 10.1109/ TDC-LA.2010.5762887

Leal G., J.L.; Albarracín, J.A. y Ortega B., H. (2009). Sistema de monitoreo SAS BEE - Un caso de integración y aplicación de nuevas tecnologías. ITECKNE, 6(2), 36-43.

Longo, M.; Roscia, M.C. y Zaninelli, D. (2015). Net zero energy of smart house design. En: 2015 International Conference on Clean Electrical Power (ICCEP) (pp. 548-554). Taormina, Italia. doi: 10.1109/ ICCEP.2015.7177544

Nedelcu, A.V.; Sandu, F.; Machedon P., M.M.; Alexandru, M. y Ogrutan, P. (2009). Wireless-based remote monitoring and control of intelligent buildings. En: IEEE International Workshop on Robotic and Sensors Environments (pp. 47-52). Lecco, Italia. doi: 10.1109/ROSE.2009.5355999

Nguyen, L.L.; Nguyen, M.T.; Kien, M.T.; Cuong, L.Q. y Nguyen, T.D. (2015). On the design of wireless access smart grid network on $868 \mathrm{MHz}$ frequency band. En: 2015 2nd National Foundation for Science and Technology Development Conference 
on Information and Computer Science (NICS) (pp. 110-114). Ho Chi Minh City, Vietnam. doi: 10.1109/NICS.2015.7302173

Pinzón C., J.D.; Santamaría P., F. y Corredor R., A. (2014). Uso racional y eficiente de la energía en edificios públicos en Colombia. Revista Científica, 19, 93-103.

Quintana, B.; Pereira, V. y Vega, C. (2015). El factor para dignificar espacios de vivienda social se encuentra en la Domótica1. Entre Ciencia e Ingeniería, 9(17), 81-89.

Tapia, A.; Bernal, C.; Rodríguez, G.; Soto, J.D. y Candelo, J.E. (2013). Energy efficiency and environmental impact of small markets in Barranquilla-Colombia. En: 2013 12th International Conference on
Environment and Electrical Engineering (EEEIC) (pp. 77-81). Wroclaw, Polonia. doi: 10.1109/ EEEIC.2013.6549593

Unidad de Planeación Minero Energética (UPME) (2015). Informe mensual de variables de generación y del mercado eléctrico colombiano - marzo de 2015. Recuperado en febrero de 2016, de: http://www.siel.gov.co/portals/0/generacion/2015/ Seguimiento_Variables_Marzo_2015.pdf

Vera R., C.A.; Barbosa J., J.E. y Pabón G., D.C. (2015). Parámetros de configuración en módulos XBEE-PRO S2B ZB para medición de variables ambientales. Revista Tecnura, 19(45), 141-157. doi: 10.14483/ udistrital.jour.tecnura.2015.3.a11 